\title{
Cognitive Rationality and Its Logic-Mathematical Language
}

\author{
Svetlana Masalova \\ Developing Qualification and Requalification of the Educational Staff Institute, Rostov-on-Don, Russia
}

\begin{abstract}
The article deals with the cognitive (flexible) rationality, combining rational and irrational moments of the scientific search of the cognizing subject. Linguo-cognitive model of the concept as the flexible regulative rationality reveals the activity of the cognitive processes and the mentality of the epistemological-ontic subject, its leading constructive role in the formation of the language, and world pictures of conceptual post-non-classic type. Logic-mathematical languages reveal "deep structures" of the subject's cognitive rationality.

Keywords: rationality, cognitive (flexible) rationality, cognizing subject, mentality, scientific search, concept, regulative
\end{abstract}

\section{Introduction}

The purpose of this paper is the analysis of the cognitive rationality and logical-mathematical language.

At the stage of the post-non-classic science, the issue of the rationality in the science remains relevant. Moreover, possible correction of the scientific rationality, understood not as an abstract-logical but as a socio-cultural, developing, and structurally complex phenomenon. Scientists name its following main forms: anarchic (P. Feyerabend), moderate (V. A. Lektorsky), descriptive (I. T. Kasavin), paradoxical (V. N. Porus), open and closed (V. S. Shvirev), special rationality (E. P. Nikitin), "hyper-rationality" (A. A. Novikov), communicative rationality (George H. Mead \& J. Habermas), value-rationality (V. G. Fedotova), etc.. But these forms of rationality are not enough to show all its potential qualities and characteristics.

Psychologists and philosophers were convinced that strictly theoretical thinking is not and cannot be the only form of the scientific thinking. It allows other rational forms: empirical thinking, common sense, and everyday awareness. Such rationality is not a rigid cognitive procedure, it allows the inclusion of the subject in the cognitive process that enhances the possibilities of the subject cognition as a carrier of the rationality.

The aspect of the rationality analysis in the article is the cognitive one. In the cognitive science, the category of the "cognitive rationality" is introduced. It is absent in the modern science, but is demanded in the cognition of the nature of the cognizing subject.

There are almost no works that address the cognitive rationality. This work examines this issue in the interdisciplinary aspect at the junction of the cognitive psychology, philosophy, cognitive linguistics, and "non-classical" mathematics.

The object of the study is: cognitive rationality as the capacity of the subject to the adequate and effective constructive scientific cognition. The subject of the study is logic-mathematical language of the cognitive rationality.

Svetlana Masalova, Ph.D., professor, Developing Qualification and Requalification of the Educational Staff Institute. 


\section{Research Issue}

The rationality issue is relevant as the issue of the mental essence of the cognizing subject. The variety of the activity forms generates a variety of forms of knowledge rationality and action rationality. This variety shows the relativity, inconsistency, and historicity of the rational, which is overcome by the dynamic development of the subject.

The carrier of the rational consciousness, implementing it in a rational activity, is the subject associated with the object of the cognition.

In the "classical rationality type", the subject-object relationships are determined by the rigid boundaries of the rationality. In the "non-classical rationality type", the system "subjects-object" is dynamic, subject and object are changed and determined, and not rigidly, but more flexibly under the influence of the chance, possibility, etc.. "Post-non-classical rationality" includes the subject in the world picture where he/she has a creative role of the "system-ogenesis core" by using the knowledge as a cognition tool. Cognition methodology includes a synergistic approach to the understanding of the world and the interpretation of it as a self-developing integrity, but being unstable, unsteady, unbalanced, chaotic, and uncertain.

The subject, using intuitively the irrational forms as a cognitive tool, reveals much diversity of acquiring new knowledge about the object, oneself, his/her cognitive abilities, and capabilities. This unaccounted "rationally irrational binarity" of the subject, manifested in the scientific cognition process at the intermediate stage of the scientific search and "wandering" along the labyrinth of the consciousness, needs to be emphasized.

Thus, the basic types and forms of the rationality are characterized by a scale of the subject cognitive activity in streamlining the knowledge of the world, forming a research methodology and organization of the corresponding activity, being adequate, constructively effective, and epistemologically relevant.

\section{Research Methods}

This research uses the following methods (approaches):

(1) Cognitive - considers the "content" of the cognition (how a person perceives and interprets the world), reveals the features of the explanation and understanding of the general principles of the mental processes management in the human brain, perception, and understanding of the world;

(2) Discursive - takes into account the dynamics of the language as a "form" of the cognition, changes that occur in the language and cognitivistic models of the language processes;

(3) Experientional - examines the language space of the subject in the direction of the movement from the linguistic to the conceptual world picture based on the experience not only of the theoretical but also of the everyday cognition;

(4) Logic-mathematical-identifies the cognitive abilities of the formalized forms of the intellectual activity of the subject.

The greatest heuristic potential has the cognitive method.

\section{Hypothesis}

We assume that: (1) The cognitive rationality due to its attribute of "flexibility" reflects the integrity and unity of the rational and irrational characteristics of the cognizing subject in its scientific activity; and (2) Logic-mathematical languages reveal "deep structure" of the cognitive rationality of the subject. 


\section{Results}

Sensual and rational levels of the cognition and biosocial nature of the man "illuminate" the existence of two differentiated sections - empirical (ontological) and epistemological in the cognizing "complex" of the subject, its "cognitive matrix". But the man - a "holistic" subject of the cognition, in his third hypostasis, synthesizes his physical-psychological, abstract-epistemological, and logic-methodological nature and form, and he obtains an existential-anthropological and socio-cultural-historical understanding.

Study of the nature of the cognizing subject that has rational and irrational forms of the cognition and identifying their unity has led to the following models.

In the "classical rationality style", the subject is averaged, it is not represented by its individual cognitive characteristics that are generally ignored in the cognitive activity, separated from the object, and independent as an "epistemological" subject. In the "non-classical rationality type", the subject and object are mutually determined. "Post-non-classical rationality" examines the subject as both empirical (ontological) and epistemological simultaneously. "Post-non-classical rationality" gives the subject the right to choose the best ways of favorable and successful interaction with the world and survive in it. Value-target embodiment of the rationality is rather diversified, its forms rapidly evolve and are intertwined. Subject is filled with the specifics close to the reality, it becomes "ontic".

The nature of the thought that has always belonged to the subject, makes determination of its content and form by the nature and specificity of the subject as its carrier. Process and result of the scientific search of the scientist depend on his/her outlook, intellectual ability, erudition, social orientation, religion, nature, temperament etc.. "Cognitive rationality"-logical cognition, includes the role of the anthropological and prelogical characteristics of the cognizing subject, its prior knowledge, methodology, and cultural and historical context of the scientific creativity (Masalova, 2006).

Cognition is a complex-integrated structure of the knowledge. It covers observable human actions, their mental representations, processes, and results of the conscious and unconscious comprehension of the world and body-motor experience, and it reveals the internal representation of the cognizing subject, a set of cognitive tools that generate these actions, including the linguistic forms of action with the knowledge.

The main attribute of the cognizing subject as a carrier of the cognitive rationality is flexibility of its consciousness and activity. "Cognitive" rationality is a "flexible" rationality of the cognizing subject, the characteristic of a productive mind. The subject has not only the ability to intellectually investigate the object, but it also reveals the cognitive capabilities of its emotional and rational sphere, including the potential ones that define the specifics and nuances of the scientific creative search. In the concept of flexible, the author singles out aspects such as consideration, vigilance, insight, depth, historical thinking, its dialectics, wisdom, high sensitivity, resonant tune to the object, etc..

Antipode of the "flexible" rationality is "rigid" rationality. The author associates it with a relatively stable set of the rules, regulations, standards of the mental and objective activity of the certain community with formal logic, classical rationality type, metaphysical way of thinking, principle of unique determination, linearity, and others. The attributes of the "rigid" rationality are dogmatism, rigor, inertia thinking, rigidity (perceptual, affective, and motivational), self-absorption, increased self-esteem, localism, stubbornness, ignoring other alternatives, etc..

The main difference between these forms of the rationality is in ways of cognition as the cognitive activity, 
understanding the nature of the cognizing subject and in the question of correlation of the object and subject. Logical cognition methods only serve as the cognition tool. The most important in the cognition is the activity of the scientist as a subject of the cognition and adequate correspondence of the process of acquiring the knowledge, specific standards of the subject's reasoning to the cognition process in general.

The subject seeks to adequately reflect in the verbal form, all interconnection nuances with the object of the cognition, to reveal in the most complete way the characteristics of the transition from the abstract to the concrete-general, taking into account the evolution of object-subject interconnection. Such cognitive rationality is flexible. It supplements "classical" (logically "rigid") scientific rationality.

The author considers the concepts of the "cognitive rationality" and the "flexible rationality" as synonyms.

Flexibility of the mind of the cognizing subject is regarded as an effective feature of the productive consciousness which is manifested:

(1) In reconstruction of the available solutions of the problem;

(2) In choosing the appropriate and effective way of cognition and activity;

(3) In discovering in the object of hidden, but cognizable characteristics;

(4) In a phased unfolding of the problem in the scientific search.

Flexible rationality is an unfolding of the mental entity of the active cognizing subject, its identity in the process of the activity. Flexibility of the consciousness and activity helps the improvement, transformation, and modernization of the solution methodology of the practical and theoretical problems.

Not flexible rationality but cognitive science has come to the conclusion that there are specific forms that characterize the consciousness of the cognizing subject.

Cognitive (flexible) rationality combines both dialectical thinking that has reached a stage of particular universality in the theoretical consciousness and synergistic thinking, showing the nonlinear stochasticity of the cognition process. Origins of the flexible rationality are evolved in other historical types of the rationality. And the flexible rationality appears as the highest form of the cognition strategy.

Structural basis of the cognitive (flexible) rationality is a concept - a form of the inseparability of the rational and irrational cognition.

Concept as a linguistic means is the universal way of transformation of the objective world into the world of the human (sense) existence. The concept is implemented in the word, the phrase, statement, and discourse. The concept is a part of the linguistic world picture as a special kind of model of the world in general. It performs the verbal and world-view functions:

(1) Linguistic world picture is constructed as a result of the speech-thought activity;

(2) Deep meanings of the cognizing subject and his/her ethnic group are expressed;

(3) Cultural level of each linguistic identity included in the concrete historical epoch is accumulated;

(4) Cultural values, relationships, ideals, ethno-psychological, and ethno-linguistic features of a particular historical time are fixed.

In the concept, the subject by reviewing the information received in the cultural and ethnic society, processes and reprocesses it, basing on the certain methodological assumptions, attitudes, and values. The concept is the objective information in the subjective vision, based on and in accordance with the mental characteristics and analytical abilities of the subject, also including the irrational forms of the cognition.

In the study of the concept and speech-thought activity of the subject, the linguists use the most delicate 
language mechanisms - juxtaposition, completion, development, and perspectivization (Babina, 2003), which is called the cognitive "linguistic nano-technologies". They allow identifying the deepest layers of the language consciousness and mental nature of the cognizing subject.

In general, the concept appears as a certain psycho-linguo-cognitive way and perspective of the prior-theoretical understanding of the reality and its modeling in the holistic vision of the world and is a regulative of the cognitive (flexible) rationality.

\section{Discussion}

The result of the cognitive activity of the subject is a system of meanings, a "cognitive matrix" that characterizes the outlook, attitude, and "world perception" world view of the cognizing subject as cognitive "tools".

Flexible rationality is "crystallized" and embodied in the certain language form (concept), which should be adequate to its content. It "pushes" the subject to a constructive discovery of a new form.

The concept is presented as a verbal dynamic model of the folk culture traditional imagery, in which the cognizing subject is immersed. The concept captures the characteristics of the concept sphere. The subject in the concept as a "bridge" connects the individual cognition with the public one, expresses by the "keywords", gives an assessment to the cultural phenomenon of the different national linguo-cultural communities, and promotes the mutual understanding in the inter-cultural communication.

The concept synthesizes the knowledge of the world, subject, and language. It is a "process" of understanding (information processing), approached from its content side, it is fundamentally "dynamic". Flexible rationality is an unfolding of the mental nature of the actively cognizing subject, and its identity is in the process of activity. In operating of the concepts, the flexible force of the cognitive rationality is expressed.

The highest level of the flexible rationality achieves the concrete universality through the appropriate logic-mathematical methods, forms, adequate to address the non-formalized tasks and it takes into the consideration: (1) nuances of the intermediate "wandering" searches that reflected the specificity of the epistemological-ontic subject; and (2) reserves of the cognitive mental activity of the subject.

Intersection (conjugation) of these circumstances leads to the creation of the "non-classical" methods because of the heuristics role of the cognitive (flexible) rationality as intuitive and strategic framework of the cognition.

Language of the cognitive (flexible) rationality can be, and is, in the author's opinion, such logic-mathematical methods, which are adequate for the certain tasks. Logic is a "deep structure" of the rationality (Griftsova, 1999). Logic-mathematical methods are inherently extremely rational and formalized language of the classical rationality. But not all aspects of the cognizing subject activity can be formalized. Where the nonstandard problems or the solution of the standard problems require the nonstandard solutions and methods, leading to a desired result, one needs other ways of the cognition and penetration into the new regularities. Hence, the need for the search of the adequate methods for the solution of the non-formalized problems includes the logic-mathematical ones.

New methods are also rational and logical. But their rationality is of a different kind: It will no longer be standard, "rigid" but the one retained, first, nuances of the intermediate "wandering" searches for the empirical subject, and secondly, the one opened reserves of the cognitive mental activity of the subject. Intersection (conjugation) of these circumstances will lead to the creation of new "non-classical" nonstandard methods 
because of the heuristics role of the flexible rationality as intuitive and strategic framework of the cognition.

To the logic-mathematical languages of the cognitive (flexible) rationality, the author attributes the "irrational" mathematics, intuitionism, Zadeh fuzzy sets, many-valued logic, etc..

The essence of the "irrational" mathematics is presented by its author V. V. Tselishev (2006). It is the ability of the mathematical practice (not theory) to reflect the intentions of the subject and control his/her use of the rational statements of the mathematical language with the ways that are unconscious at any given moment and that are effective in practice without the theoretical, especially philosophical explanation.

"Intuitionism" in mathematics "caught" the idea of the flexible rationality before then the various fields of the natural science. The study was carried out within the abstraction of the potential (becoming) infinity. Intuitionism revealed the constructive possibilities of the mathematician as a creative subject and his/her ability to create a flexible cognition methodology, to include the moments of the irrational (intuition) into the rational scientific search. Mathematical theory was based on a specially designed principles and methods by the subject. The law of the excluded middle was not rejected. The definite limits of its applicability changed: It is used in the operations with the finite domains of the objects and does not apply to the operations involving the infinite aggregate in the process of growth. Thus, the subject from the state of the theoretical uncertainty comes not only to the particular theoretical (mathematical), but also philosophical and methodological certainty. Mathematics from the mathematics of the "existence" acquires the character of the "composition". This idea of intuitionism was subsequently developed by the non-classical epistemological paradigm.

As a language of the cognitive (flexible) rationality can be considered as the "fuzzy" sets logic by Zadeh (1965), in which the facts of the semantic boundaries fuzziness allow the logical interpretation by adapting the Boolean algebra. The notion of the continuous membership function with values on the interval from 0 to 1 is introduced instead of the discrete concept of the element membership to a certain category. "Flexible" semantics is opposed as the antipode to the constraints' semantics, as for the first time on the basis of the principle analysis of the fuzzy logic, Lakoff (1973) introduced it to the linguists. Thanks to its flexible constructive logical characteristics, the fuzzy sets theory has advantages over "natural logic" or a value theory in describing the impact of the certain words-operators (Hersch \& Caramazza, 1976); value theory is based on the harsh attribution of the characteristics, and fuzzy sets theory on the combinatorial semantics of the assessment (approximate) quantifiers. McCawley (1978) believed that Zadeh's analysis cannot reveal the sources of the semantic anomalies. The analysis based on "fuzzy logic" does not separate adequately the communicative implications from the conditions of the true statements with the fuzzy semantics. However, although the axioms of the fuzzy sets logic have not been confirmed entirety, the fuzzy sets theory is a promising basis to describe the combinatorial semantics of the lexical items with a fuzzy value.

"Many-valued" logic that we consider as the language of the flexible rationality is potentially applicable at lexical graphic description of the two types of "constraints": those that relate to the degree of the truth, and those that produce or suppress various measurements of the truth. In many-valued logic many values of truth are allowed. Thus, Lukasewich (1959), who at first developed three-valued logic, introduced as the third value of the true statement, the value expressed by the words "possible", "neutral", etc.. Later, Lukasewich (1959) developed the system of modal logic, studying the logic operations with the statements expressing the values "possible", "impossible", and so on, as well as the system of four-valued and infinitely-valued logics. He has also offered a "bracket-free symbolic", departing from the graphic writing of the logical formulas and moderating the formalism. E. L. Post built a system of "n-valued logic", in which to the statements are 
attributed any finite and infinite set of the truth values, thus extending and expanding the studies, conducted by the methods of the social-humanitarian cognition in understanding the nature of the cognizing subject. It is presented as a holistic ("epistemological-ontic") subject with its prelogical and anthropological characteristics, expressing doubt, uncertainty, and other irrational forms of cognition in the scientific creative work. All the nuances of the "wandering" search for the truth by the cognizing subject, the many-valued logic reflects by the respective categories: "probability", "opportunity", "incredibility", "impossibility", etc.. Thus, the conceptual apparatus of many-valued logic confirms its status introduced by us as the language of the cognitive (flexible) rationality as a new methodology of the scientific cognition. Of course, many-valued logic is not limited by the introduction of these categories, it solves its main issue to determine the truth or falsity of the judgments.

\section{Conclusions}

Scientific rationality, by changing from the classical type through the non-classical to the post-non-classical type, changed its language and its form.

"Classical rationality paradigm" used such forms as: perception, idea, concept, hypothesis, and theory. But cognition is not limited to these traditional forms.

"Post-non-classical rationality paradigm": There are no pure forms of the rationality in the nature, there are mixed forms - on the borderline of the sensory-experienced and rational cognition. To the new form of the rationality, the author attributes the "cognitive rationality"-logical cognition based on prelogical and anthropological presuppositions of the scientific activity. Cognizing subject is presented as the "epistemological-ontic subject" carrier of rationality ("epistemological" subjects) and carrier of irrational ("ontic" subject): feelings, emotions, desires, moods, intuition, faith, doubt, will, etc. (see Figure 1).

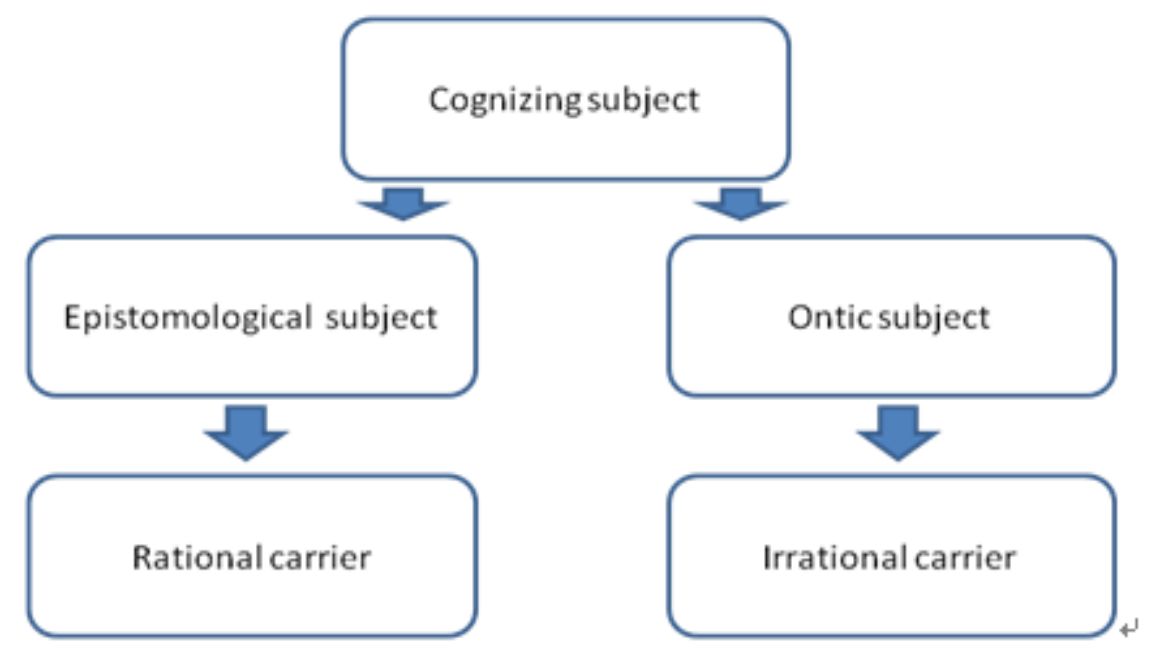

Figure 1. Structure of cognizing subject.

Hypothesis 1 was confirmed.

"Cognitive" rationality is a "flexible" rationality of the cognizing subject, characteristic of the productive consciousness that is manifested in the reorganization of the existing solutions to the problem that the subject has for a more efficient and optimal. Operating on the concepts is a flexible rationality.

Flexible rationality is embodied in the certain linguistic form, which should be adequate to its content.

Language of the flexible rationality is formed by the new modern non-standard logical and mathematical 
methods, their development and improvement will allow developing a coherent and complete concept in the future. Thanks to their flexible constructive logical properties, the formalized languages reveal "deep structures" of the cognitive rationality of the subject. Hypothesis 2 was confirmed.

Cognitive (flexible) rationality is the highest form of the subject's cognition strategy at the stage of post-non-classical science.

\section{References}

Babina, L. V. (2003). Cognitive bases of the secondary phenomena in language and speech. Monograph: Tambov-Moscow. Chalmers, D. (1996). The conscious mind: In search of a fundamental theory. N.Y.

Griftsova, I. N. (1999). Logic as a theoretical and practical discipline: On issue of correlation between formal and informal logic. M.

Hersch, H., \& Caramazza, A. (1976). A fuzzy set approach to modifiers and vagueness in natural language. Journal of Experimental Psychology: General, 105(3), 254-276.

Lakoff, G. (1973). Hedges: A study in meaning criteria and the logic of fuzzy concepts. Journal of Philosophical Logic, 2(4), 458-508.

Lukasewich, J. (1959). Elements of mathematical logic. N. Y.

Masalova, S. (2006). Philosophical concepts as regulatives of flexible rationality: Transformation from antiquity to modern times. Monograph: Rostov-on-Don.

McCawley, J. D. (1978). Logic and the lexicon. In Papers from the Parasession on the lexicon (pp. 261-277). Chicago: Chicago Linguistic Society.

Post, E. L. (1921). Introduction to a general theory of elementary propositions. American Journal of Mathematics, 43, 13.

Tselishev, V. V. (2006). Is irrational mathematics possible? Retrieved from http://www. philosophy.nsc.ru

Zadeh, L. A. (1965). Fuzzy sets: Information and control (pp. 338-353). 